\title{
Electrocardiogram in anterior mid-ventricular Takotsubo syndrome variant
}

\author{
John E. Madias ${ }^{1,2, *}$ \\ ${ }^{1}$ Icahn School of Medicine at Mount Sinai, New York, NY, USA; \\ ${ }^{2}$ Division of Cardiology, Elmhurst Hospital Center, Elmhurst, NY, USA.
}

To the Editor:

I enjoyed reading the report by Demirelli et al., published in the May, 2015 issue of the Intractable \& Rare Diseases Research, pertaining to a 59 year old woman with emotional stress-induced anterior midventricular Takotsubo syndrome (TTS) variant (1). The authors described the electrocardiogram (ECG) as showing T-wave inversions (TWI) in leads V1V4. A recent paper reported an ECG correlate of TTS consisting of transient low voltage QRS (LVQRS) complexes (2). These ECG changes have been attributed to TTS-induced myocardial edema (ME), as detected by cardiac magnetic resonance imaging (cMRI) (2). Also TWI has been attributed to apicobasal ME gradient, as detected by cMRI (3). The present patient had a cMRI 4 weeks after her discharge, which confirmed the absence of chronic scar changes, compatible with TTS, but such late cMRI would not be suitable for the detection of ME, an early feature of TTS. Transient LVQRS often impacts the limb ECG leads (2), while affects leads I and aVL in patients with the midventricular TTS variant $(4,5)$. Did this patient show transient LVQRS in the V1-V4 leads, which showed TWI? Also if serial ECGs were recorded, and if there was an ECG of this patient recorded prior to her admission with TTS, and at her 4 week follow-up, were there any transient LVQRS ECG changes, and in which leads?

\section{References}

1. Demirelli S, Ermis E, Hatem E, Uslu A, Askin L. Focal mid-ventricular anterior ballooning: An unusual pattern of Takotsubo cardiomyopathy. Intractable Rare Dis Res. 2015; 4:108-110.

Released online in J-STAGE as advance publication June 23, 2015.

*Address correspondence to:

John E. Madias, MD, FACC, FAHA, Division of Cardiology, Elmhurst Hospital Center, 79-01 Broadway, Elmhurst, NY 11373, USA.

E-mail:madiasj@nychhc.org
2. Madias JE. Transient attenuation of the amplitude of the QRS complexes in the diagnosis of Takotsubo syndrome. Eur Heart J Acute Cardiovasc Care. 2014; 3:28-36.

3. Perazzolo Marra M, Zorzi A, Corbetti F, De Lazzari M, Migliore F, Tona F, Tarantini G, Iliceto S, Corrado D. Apicobasal gradient of left ventricular myocardial edema underlies transientT-wave inversion and QT interval prolongation (Wellens' ECG pattern) in Tako-Tsubo cardiomyopathy. Heart Rhythm. 2013; 10:70-77.

4. Madias JE. Electrocardiogram lead-specific QRS attenuation in an atypical midventricular case of takotsubo syndrome. J. Electrocardiol. 2013; 46:728-729.

5. Madias JE. Is the electrocardiogram different in recurrent episodes of Takotsubo syndrome involving 2 different left ventricular territories in the same patient? Int $\mathrm{J}$ Cardiol. 2015; 182:271.

(Received May 22, 2015; Accepted June 1, 2015) 